\title{
Limits on the diffuse flux of ultra-high energy neutrinos using the Pierre Auger Observatory
}

\author{
Sergio Pastor ${ }^{\mathrm{a}, 1}$, for the Pierre Auger Collaboration ${ }^{\mathrm{b}}$ \\ ${ }^{a}$ Institut de Física Corpuscular (CSIC-Universitat de València), Ed. Institutos de Investigación, Apartado 22085, 46071 Valencia, Spain \\ ${ }^{b}$ Av. San Martín Norte 304 (5613) Malargüe, Prov. de Mendoza, Argentina
}

\begin{abstract}
The surface detector array of the Pierre Auger Observatory is sensitive to ultra-high energy neutrinos in the cosmic radiation. These particles can interact close to ground, both through charged and neutral currents in the atmosphere (down-going) and, for tau neutrinos, through the Earth-skimming mechanism (up-going) where a tau lepton is produced in the Earth crust that can emerge and decay in the atmosphere. Both types of neutrino induced-events produce an inclined shower that can be identified by the presence of a broad time structure of signals in the water-Cherenkov detectors. Using data collected from the surface detector array of the Pierre Auger Observatory, we present the corresponding limits on the diffuse flux of ultra-high energy neutrinos.
\end{abstract}

Keywords: Pierre Auger Observatory, Ultra-high energy neutrinos

\section{Introduction}

The existence of cosmic neutrinos with energies in the $\mathrm{EeV}$ range and above is required by the observation of ultra-high energy cosmic rays (UHECRs). Although the nature of the very energetic cosmic radiation and its production mechanisms are still uncertain [1,2], all models of UHECRs predict neutrino fluxes from the decay of charged pions, produced either in interactions of the cosmic rays in their sources, or in their subsequent interactions with background radiation fields. The socalled cosmogenic or GZK neutrinos [3] are produced in the interaction of UHE protons with the cosmic microwave background (CMB). This flux of cosmogenic neutrinos is to some extent uncertain since it depends on the composition of primary UHECRs and on the nature, cosmological evolution and spatial distribution of the sources (see e.g. [4]).

The observation of UHE neutrinos would open a new window to the universe, since they can give information on regions that are otherwise hidden from observation by large amounts of matter in the field of view. Moreover, UHE neutrinos travel unaffected by magnetic

Email address: pastor@ific.uv.es (Sergio Pastor)

${ }^{1}$ Work supported by the Spanish grants FPA2008-00319 and CSD2009-00064 (MICINN) and PROMETEO/2009/091 (Generalitat Valenciana)

\section{2. Detection of UHE neutrinos with the Pierre Auger Observatory}

Neutrinos, even at very high energies, present a low interaction probability which means that a large amount of matter is needed to detect these elusive particles. In the case of UHE cosmic neutrinos, the Earth atmosphere is the target where the primary particles inter-

September 28,2010

fields and, hence, they essentially maintain the direction of their production places. The detection of very energetic cosmic neutrinos is the aim of many experiments which employ different techniques, from neutrino telescopes such as IceCube or Antares to experiments like ANITA, that searches for radio waves from tion techniques is based on the observation of extensive air showers (EAS) in the atmosphere initiated by UHE neutrinos, which could be detectable by a large ground

In this contribution, we describe the sensitivity of the surface detector array of the Pierre Auger Observatory to UHE neutrinos with energies around $\mathrm{EeV}$ and larger. explain the identification criteria used to distinguish CRs, such as protons or heavy nuclei. The analysis of Auger data reveals no neutrino candidates, leading to stringent limits on the diffuse flux of UHE neutrinos. 


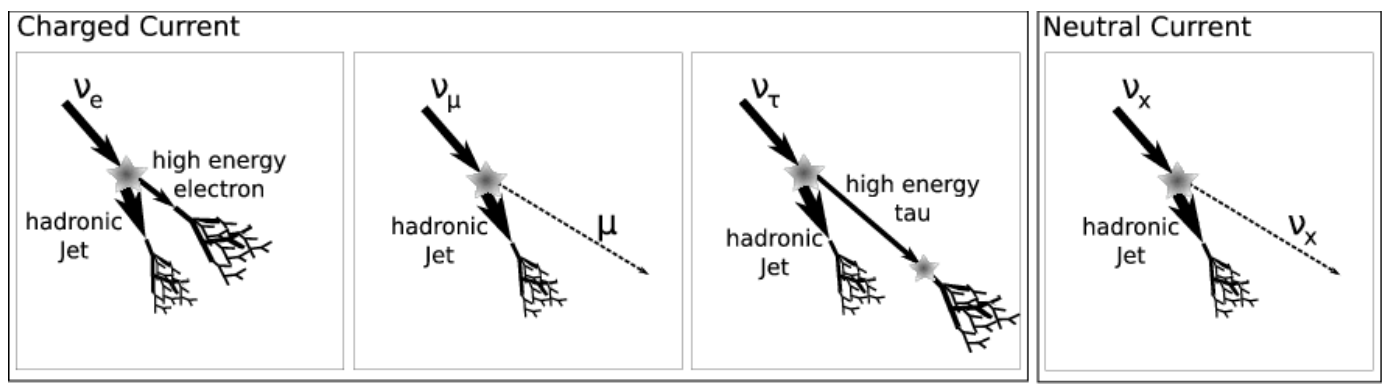

Figure 1: Types of neutrino interactions that initiate particle showers in the atmosphere. In neutral current (NC) processes the scattered neutrino carries away a large fraction of the primary energy and only part is transferred to the shower. A similar case is that of charged current (CC) interactions involving UHE $v_{\mu}$ 's, where the outgoing muon usually decays under the ground and does not produce an EAS. Instead, the emerging charged leptons essentially carry all the initial energy in CC processes with a $v_{e}$ or a $v_{\tau}$, with the possibility of a double-bang shower if the $\tau$ travels a long distance before decaying.

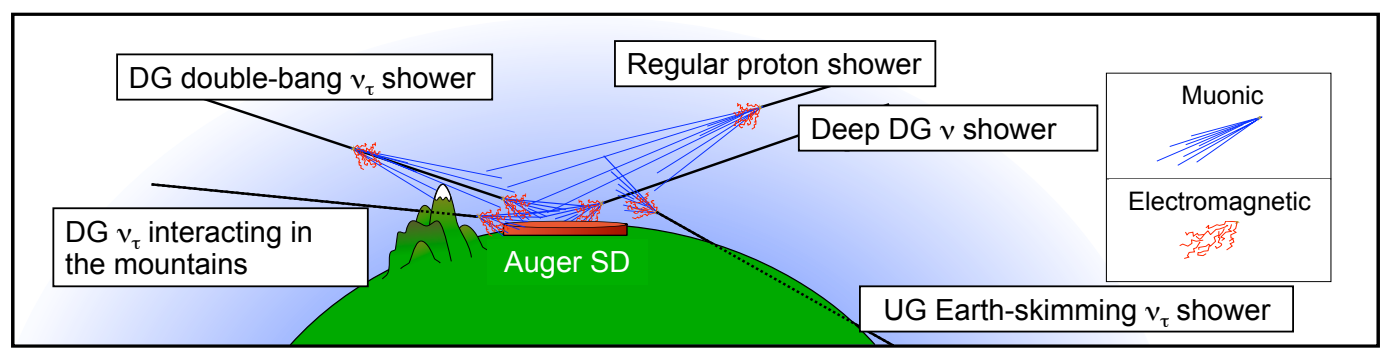

Figure 2: Simplified picture of the different types of particle showers induced by UHE neutrinos compared to proton-induced showers. The surface detector of the Pierre Auger Observatory is sensitive to both up-going (UG) or Earth-skimming tau neutrinos and down-going neutrinos (DG) of all flavours.

act producing an EAS that can be detected in experiments such as the Pierre Auger Observatory. These are the so-called down-going (DG) events, where neutrinos of all flavours interact at any atmopheric depth through charged or neutral currents, as shown in Figure 1, and develop an EAS. Instead, only UHE tau neutrinos can lead to up-going (UG) or Earth-skimming events in an efficient way, when they interact in the Earth crust and produce a tau lepton that can emerge and decay in the atmosphere. If the decay of such tau leptons occurs in flight over the detector array, they may initiate detectable air showers $[5,6]$. Tau neutrinos are expected to be suppressed in the production processes, because they do not appear in the charged pion decay chain. However, the effect of neutrino flavour oscillations over cosmological distances modifies the initial composition and leads to approximately equal fluxes for all flavours. DG and UG neutrino-induced showers are depicted in Figure 2.

The main background for the detection of EAS induced by UHE neutrinos is the particle showers initiated by UHECRs: protons or heavy nuclei and possi- bly photons. UHECRs interact high in the atmosphere, producing particle showers that contain muons and an electromagnetic component of electron, positrons and photons. This latter component reaches a maximum at an atmospheric depth of order $800 \mathrm{~g} \mathrm{~cm}^{-2}$, extinguishing gradually within the next $1000 \mathrm{~g} \mathrm{~cm}^{-2}$. Thus after roughly a couple of vertical atmospheric depths only high energy muons survive. In the first stages of development, while the electromagnetic component develops, the time spread of the particles in the shower front is large $(\sim \mu \mathrm{s})$. When the shower becomes old, most of the particles in the shower front, the high energy muons, arrive in a short time window ( $\sim 100 \mathrm{~ns})$. As a consequence very inclined showers induced by UHECRs in the upper atmosphere reach the ground as a thin and flat front of muons accompanied by an electromagnetic halo, which is produced by bremsstrahlung, pair production, and muon decays, and has a time structure very similar to that of the muons. On the other hand, if a shower is induced by a particle that interacts deep in the atmosphere (a deep neutrino interaction in air, or a tau decay), its electromagnetic component could hit 
the ground and give a distinct broad signal in time. The panels in Figure 3 represent these various possibilities.

The Pierre Auger Observatory [7] has been designed to measure UHECRs with unprecedented precision. It employs the two available techniques to detect EAS, namely, arrays of surface particle detectors and telescopes that detect fluorescence radiation. The surface detector array (SD) of the Southern Auger Observatory, recently completed in the Mendoza province (Argentina), consists of 1600 water Cherenkov tanks arranged in a hexagonal grid of $1.5 \mathrm{~km}$ that covers an effective area of $3000 \mathrm{~km}^{2}$. Each cylindrical tank of $10 \mathrm{~m}^{2}$ surface contains purified water, $1.2 \mathrm{~m}$ deep, and is instrumented with three 9" photomultiplier tubes (PMT) sampled by $40 \mathrm{MHz}$ Flash Analog Digital Converters (FADCs). Each tank is regularly monitored and calibrated in units of vertical equivalent muon (VEM) corresponding to the signal produced by a muon traversing the tank vertically [8].

The signal in each station of the Auger SD is digitized using FADCs with a 25 ns time resolution, which allows unambiguous distinction between the narrow signals induced by muons and the broad signals induced by the electromagnetic component. Thus the time structure and shape of the FADC traces, characterized by several observables [9], can help us to discriminate stations hit by an EAS in the early stages of development or by an old EAS. A set of conditions has been designed and optimized to select showers induced by UHE neutrinos, ei- ${ }^{143}$ ther UG or DG events, rejecting those induced by UHE- 144 CRs. These conditions constitute the neutrino identifi- 145 cation criteria for SD events, described in the next sec- 146 tion. The fluorescence detectors can also be used for 147 neutrino searches but the nominal $10 \%$ duty cycle of 148 this technique reduces the sensitivity. Here only the data 149 collected with the SD of the Pierre Auger Observatory 150 is used to search for UHE neutrinos.

\section{Neutrino identification criteria}

A large set of simulations of UHE neutrinos forced to 155 interact deep in the atmosphere were produced in order 156 to characterize the signal that their induced EAS would 157 produce at the SD array. The first interaction of the pri- 158 mary neutrino in the air, either NC or CC, was simulated 159 using HERWIG [10], while the AIRES code [11] was 160 used for the EAS development. For $v_{\tau}$-induced showers, the TAUOLA package [12] was used to simulate ${ }_{161}$ the $\tau$ decay and obtain the secondary particles and their ${ }_{162}$ energies.

Shower simulations were performed including the ge- 164 ographic conditions of the Auger site (e.g. geomag- 165

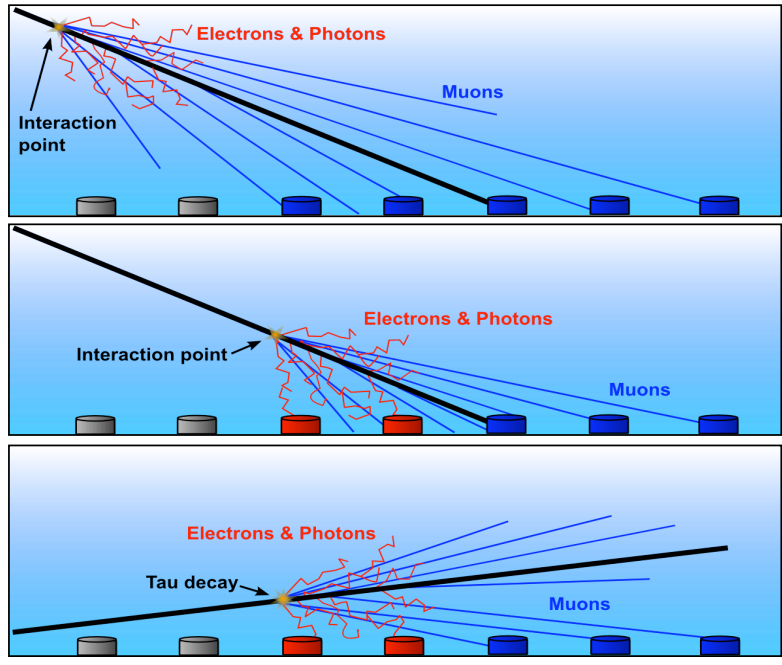

Figure 3: Upper panel: an inclined EAS induced by a proton interacting high in the atmosphere. The electromagnetic component is absorbed and only the muons reach the array of ground detectors. Middle panel: a primary UHE neutrino can initiate a deep inclined shower whose early region has a significant electromagnetic component at the detector level. Lower panel: a "young" shower can be also produced by an up-going tau lepton produced by the interaction of an Earth-skimming neutrino.

netic field) for different zenith angles. For UG showers, primary energies ranged from $10^{17}$ to $3 \times 10^{20} \mathrm{eV}$ at zenith angles between $90.1^{\circ}$ and $95.9^{\circ}$ and at an altitude of the decay point above the Pierre Auger Observatory up to $2500 \mathrm{~m}$. In this case we also simulate the propagation of up-going tau neutrinos through the Earth crust. In the case of DG neutrinos, simulations were performed at zenith angles $\theta=75^{\circ}, 80^{\circ}, 85^{\circ}, 87^{\circ}, 88^{\circ}$ and $89^{\circ}$ and random azimuth angles between $0^{\circ}$ and $360^{\circ}$ and different hadronic models. The primary neutrinos were forced to interact at different slant depths measured from the ground up to a maximum value depending on $\theta$. Finally the response of the SD array is simulated in detail using the Offline simulation package [13]. The two sets of Monte Carlo (MC) neutrino simulations were used to estimate the expected neutrino signal and the detection efficiency for both UG and DG events.

The main criterion to identify young and very inclined showers consists of looking for broad time signals in the SD stations. Two different sets of identification conditions were designed to search for UG and DG neutrinos. 

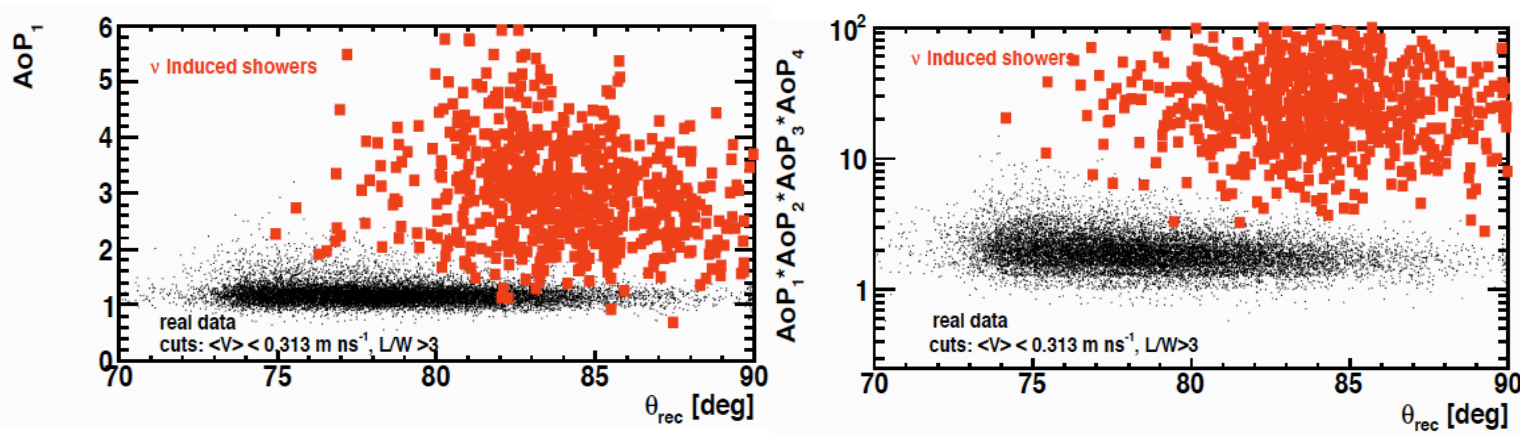

Figure 4: Distribution of the values of the area over peak for the first triggering station ( $\mathrm{AoP}_{1}$, left) and the product of AoP of the first four triggering stations (right), as a function of the zenith angle for real data and $\mathrm{MC}$ simulated neutrinos.
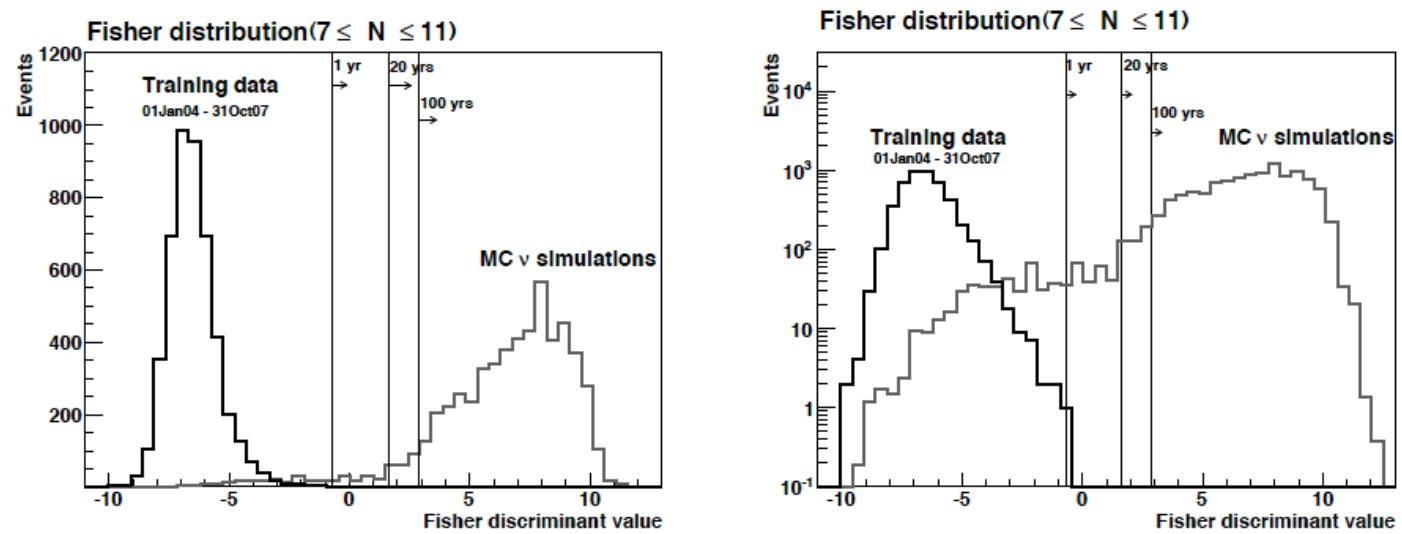

Figure 5: Distribution of the Fisher discriminant in linear (left) and logaritmic (right) scale for real Auger data in the training period (1 Jan 2004 31 Oct 2007) and MC simulated down-going neutrinos for events with multiplicity $7 \leq N \leq 11$.

\subsection{Up-going neutrinos}

Young showers are expected to trigger detector stations with broad signals releasing a so-called Time Over ${ }^{180}$ Threshold (ToT) trigger [14]. Counting ToTs stations ${ }_{187}$ can help identifying young showers, and a cut in the 188 value of the area of the signal over its peak (AoP, where ${ }_{189}$ the peak corresponds to the maximum measured current 190 of recorded trace at a single water-Cherenkov detector) 191 is applied to reject accidental muons hitting a station 192 that could mimic a ToT local trigger. After trace clean- 193 ing, very inclined showers are identified with the elon- 194 gation of their footprint, defined by the ratio of length 195 (L) over width $(\mathrm{W})$ of the shower pattern on ground, 196 requiring $L / W>5$, and the mean apparent velocity 197 $\langle V\rangle$, expected to be compatible with the speed of light 198 for quasi-horizontal showers, in the range $(0.29,0.31) \mathrm{m} \quad 199$ $\mathrm{ns}^{-1}$ with an r.m.s. scatter below $0.08 \mathrm{~m} \mathrm{~ns}^{-1}$. Finally 200 compact configurations of selected ToTs complete the 201 expected picture of young $v_{\tau}$-induced shower footprints. 202

\subsection{Down-going neutrinos}

DG neutrino events are also young and inclined showers, but the wider range of zenith angles requires identification criteria different from those applied to UG neutrinos, as described in [17]. For this purpose data collected with the Auger SD between 1 Jan 2004 and 31 Oct 2007 (about 1.2 years of the full SD array) was used as "training" data. Showers that trigger the SD array but arrived during periods with instabilities in data acquisition were excluded. After that the FADC traces are cleaned to remove segments that are due to accidental muons not belonging to the shower but arriving close in time with the shower front. Moreover, if 2 or more segments with areas comparable to each other appear in a trace the station is classified as ambiguous and it is not used. Then a selection of the stations actually belonging to the event is done based on space-time compatibility 
among them. Events with less than 4 tanks passing the 255 level 2 trigger algorithm [7] are rejected. This sample is then searched for inclined events requiring that the triggered tanks have elongated patterns on the ground, with a cut $L / W>3$. The average speed $\langle V\rangle$ measured be- 257 tween pairs of triggered stations is required to be com- 258 patible with that expected in a simple planar model of 259 the shower front in an inclined event with $\theta \geq 75^{\circ}$, al- 260 lowing for some spread due to fluctuations $(\langle V\rangle \leq 0.313261$ $\left.\mathrm{m} \mathrm{ns}^{-1}\right)$. Only events with reconstructed zenith angle 262 $\theta \geq 75^{\circ}$ are selected. Exactly the same set of conditions 263 is applied to the simulated neutrinos.

The sample of inclined events is searched for young 265 showers using observables characterizing the time du- 266 ration of the FADC traces in the early region of the 267 event. To optimize their discrimination power we ap- 268 plied the Fisher discriminant method [18] to the training 269 data, dominated by nucleonic showers, and to the Monte 270 Carlo (MC) simulations that are exclusively composed 271 of neutrino-induced showers. Given two populations 272 of events - nucleonic inclined showers and $v$-induced 273 showers in our case - characterized by a set of ob- 274 servables, the Fisher method produces a linear combi- 275 nation of the various observables (the Fisher discrimi- 276 nant $f$ ) so that the separation between the means of $f{ }_{277}$ in the two samples is maximized, while the quadratic 278 sum of the r.m.s. of $f$ in each of them is minimized. 279 Since events with a large number of tanks or multiplic- 280 ity $N$ are different from events with small multiplic- 281 ity the sample of training data is divided into 3 sub- 282 samples corresponding to events with number of tanks 283 $4 \leq N \leq 6,7 \leq N \leq 11$ and $N \geq 12$, and a Fisher dis- 284 criminant is obtained using each of the sub-samples as 285 training data. We use 10 discriminant variables of the 286 Fisher estimator: the AoP and its square of the first 4287 tanks in each event, their product, and a global early- 288 late asymmetry. In Figure 4 the distributions of two of 289 these discriminant variables are shown as a function of 290 the zenith angle for reutrino simulated showers and real 291 inclined events. One can see a clear separation between 292 the two samples.

In Figure 5 we present the distribution of the Fisher 294 discriminant for the training data and DG neutrino sim- 295 ulations with multiplicity $7 \leq N \leq 11$. Again both sam- 296 ples are well separated. The expected number of back- 297 ground events can be computed by extrapolating the ex- 298 ponential tail of the distribution of the data. This allows 299 us to find a cut-off value $f_{\text {cut }}$ for each of the sub-samples, 300 so that we expect less than one background event every 301 20 years above its value. Events with $f>f_{\text {cut }}$ are con- 302 sidered to be neutrino candidates. These cuts reject all ${ }_{303}$ real events in the training data samples while keeping a 304 significant fraction of the MC neutrino simulations [17].

\section{Exposure and limits on UHE neutrinos}

Auger data have been analyzed to look for candidate events that fulfilled the selection criteria for Earthskimming UHE tau neutrinos, as described in [15, 16]. This analysis has been updated with data equivalent to 2 years of the full Auger SD in [9, 17], where we also describe how we have applied the selection procedure and values of $f_{\text {cut }}$ for the identification of DG neutrino events to real data from 1 Nov 2007 to 28 Feb 2009 ( $\sim 0.8$ years of the full SD array), i.e. after the training period mentioned above.

Over the period analyzed, no candidate events were found for either UG or DG neutrinos. Based on this, the Pierre Auger Observatory data can be used to place the corresponding limits on the diffuse flux of UHE neutrinos. For this purpose the total exposure of the Auger SD must be evaluated, which involves folding the SD array aperture with the interaction probability and the identification efficiency $\epsilon$, and integrating in time taking into account changes in the array configuration due to the installation of new stations and instabilities in data taking.

For both UG and DG neutrinos, the identification efficiency depends on the position of the shower in the surface covered by the array and the time through the instantaneous configuration of the array. The efficiency for UG neutrinos is also a function of the $\tau$ energy and the altitude above ground of the central part of the shower $h_{c}$ (defined at $10 \mathrm{~km}$ after the decay point [19]). Instead, for DG neutrinos $\epsilon$ depends on the primary energy and the depth along the atmosphere at which the neutrino interacts, as well as on the neutrino flavour and type of interaction (CC or NC), since the different combinations of both induce different type of showers. The efficiencies for each case were obtained through MC simulations of the development of the shower in the atmosphere and the simulation of the surface detector array.

The Auger exposure to UHE neutrinos was calculated using purely MC techniques and also integrating the neutrino identification efficiencies $\epsilon$ over the whole parameter space, for the details see $[9,15,16,17]$. Several sources of systematic uncertainties have been taken into account and their effect on the exposure evaluated. Here we do not include the full list, but we note that the main systematic uncertainty for UG neutrinos is the calculation of $\tau$ energy losses [16], while for DG the dominating source is the neutrino cross section.

Once the exposure has been calculated, a limit on the flux of UHE neutrinos can be obtained assuming a 


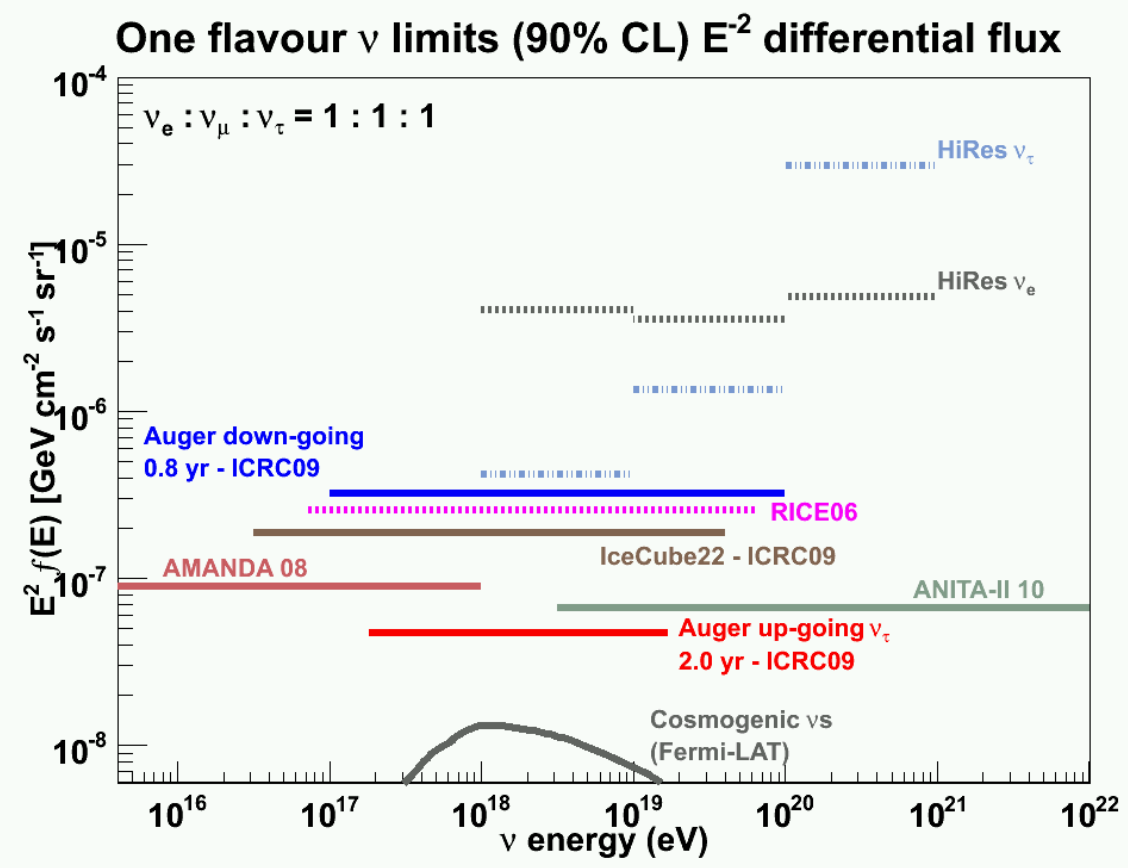

Figure 6: Integrated upper limits (90\% C.L.) from the Pierre Auger Observatory on the diffuse flux of UHE neutrinos from the analysis of the equivalent of $2(0.8)$ years of the full SD array for UG (DG) neutrinos, together with limits from other experiments. For comparison, a computation of the flux of cosmogenic neutrinos from Ref. [26] is included.

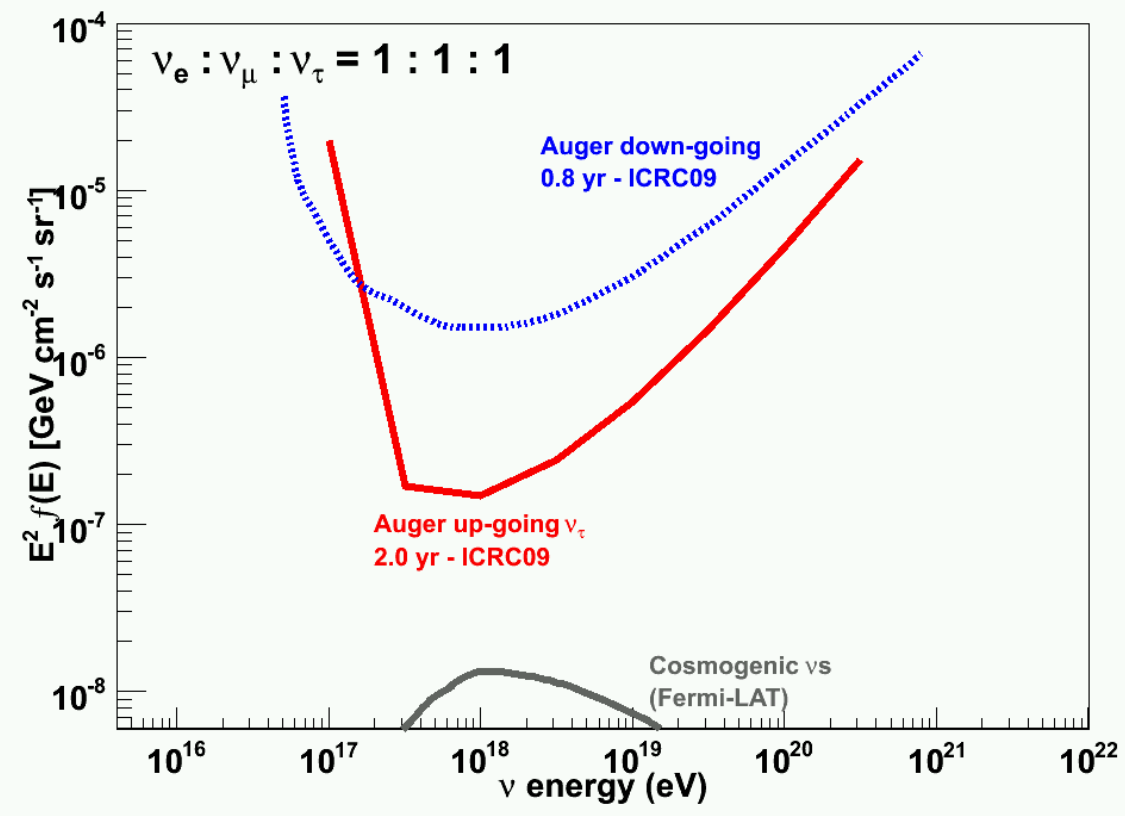

Figure 7: Differential upper limits (90\% C.L.) on the diffuse flux of UHE neutrinos from the same Auger data as in the previous figure. The sensitivity of the Pierre Auger Observatory peaks at EeV neutrino energies. 
known shape. For a $f(E)=k \cdot E^{-2}$ differential neu- 351 trino flux we have obtained a $90 \%$ C.L. limit on the all- 352 flavour neutrino flux using DG showers [17]

$$
k<3.2 \times 10^{-7} \mathrm{GeV} \mathrm{cm}^{-2} \mathrm{~s}^{-1} \mathrm{sr}^{-1} .
$$

The corresponding limit on Earth-skimming UG neutrinos is

$$
k<4.7 \times 10^{-8} \mathrm{GeV} \mathrm{cm}^{-2} \mathrm{~s}^{-1} \mathrm{sr}^{-1},
$$

which updates the limits published in $[15,16]$. It is 363 worth to mention that the topography around the South- ${ }^{364}$ ern Site of the Pierre Auger Observatory enhances the ${ }^{365}$ flux of secondary tau leptons and could improve the ${ }_{367}$ above limit up to a factor of $\sim 20 \%$.

In Figure 6 the two Auger limits on the integrated UHE neutrino flux are shown, compared with the bounds from other experiments: AMANDA-IceCube 372 [20, 21], RICE [22], HiRes [23, 24] and ANITA-II [25]. ${ }^{373}$ Finally, we include in Figure 7 the Auger limits in differential format in order to emphasize the range in energies at which the sensitivity of the Pierre Auger Observatory to UHE neutrinos peaks. These differential limits were calculated as $2.44 / E_{\nu} \varepsilon\left(E_{v}\right)$, where $\varepsilon\left(E_{v}\right)$ is the exposure. In both figures the gray line corresponds to one example of the expected cosmogenic neutrino flux, computed in [26] and consistent with HiRes and FermiLAT measurements.

\section{Conclusions}

The surface detector array of the Pierre Auger Observatory is sensitive to the EAS initiated by UHE neutrinos in the atmosphere, either down-going or Earthskimming neutrinos. We have shown how using MC simulations and training data the identification criteria for UHE neutrinos can be found. The data collected by the Auger SD has been used to present upper limits to the diffuse flux of UHE neutrinos, providing at present the most sensitive bound on neutrinos at EeV energies, which is the most relevant energy to explore the predicted fluxes of cosmogenic neutrinos. The Pierre Auger Observatory will keep taking data for about 20 years over which the bound will improve significantly if no neutrino candidate is found.

\section{References}

[1] F. Halzen and D. Hooper, Rep. Prog. Phys. 65 (2002) 1025.

[2] P. Bhattacharjee and G. Sigl, Phys. Rep. 327 (2000) 109.

[3] V.S. Berezinsky et al. Phys. Lett. B 28 (1969) 423.

[4] L.A. Anchordoqui et al., Phys. Rev. D 76 (2007) 123008.

[5] D. Fargion, Astrophys. J. 570 (2002) 909.
[6] A. Letessier-Selvon, AIP Conf. Proc. 566 (2001) 157.

[7] J. Abraham et al. [Pierre Auger Coll.], Nucl. Instr. and Meth. A 523 (2004) 50.

[8] X. Bertou et al. [Pierre Auger Coll.], Nucl. Instr. and Meth. A 568 (2006) 839.

[9] D. Góra [Pierre Auger Coll.], Proceedings of the $31^{\text {st }}$ Int. Cosmic Ray Conference 2009, Łódź, Poland.

[10] G. Corcella et al., JHEP 01 (2001) 010.

[11] S. Sciutto, http://www.fisica.unlo.edu.ar/auger/aires/

[12] S. Jadach et al., Comput. Phys. Commun. 76 (1993) 361.

[13] S. Argiro et al., Nucl. Instrum. Meth. A 580 (2007) 1485.

[14] P. Billoir and O. Blanch Bigas, Nucl. Phys. Proc. Suppl. 168 (2007) 225.

[15] J. Abraham et al. [Pierre Auger Coll.], Phys. Rev. Lett. 100 (2008) 211101

[16] J. Abraham et al. [Pierre Auger Coll.], Phys. Rev. D 79 (2009) 102001.

[17] J. Tiffenberg [Pierre Auger Coll.], Proceedings of the $31^{\text {st }}$ Int. Cosmic Ray Conference 2009, Łódź, Poland.

[18] R. Fisher, Annals Eugen. 7 (1936) 179.

[19] X. Bertou et al., Astropart. Phys. 17 (2002) 183.

[20] M. Ackermann et al. [IceCube Coll.], Astrophys. J. 675 (2008) 1014.

[21] R. Abbasi et al. [IceCube Coll.], preprint arXiv:1009.1442

[22] I. Kravchenko et al. [Rice Coll.], Phys. Rev. D 73 (2006) 082002.

[23] K. Martens [HiRes Coll.], preprint arXiv:0707.4417

[24] R.U. Abbasi et al. [HiRes Coll.], Astrophys. J. 684 (2008) 790

[25] P.W. Gorham et al. [ANITA Coll.], Phys. Rev. D 82 (2010) 022004.

[26] M. Ahlers et al., Astropart. Phys. 34 (2010) 106 